QUANTUM PROBABILITY

BANACH CENTER PUBLICATIONS, VOLUME 73

INSTITUTE OF MATHEMATICS

POLISH ACADEMY OF SCIENCES

WARSZAWA 2006

\title{
ON THE CURVATURE OF THE SPACE OF QUBITS
}

\author{
ATTILA ANDAI \\ Department for Mathematical Analysis, Budapest University of Technology and Economics \\ Egry J. u. 1, 1111 Budapest, Hungary \\ E-mail:andaia@math.bme.hu
}

\begin{abstract}
The Fisher informational metric is unique in some sense (it is the only Markovian monotone distance) in the classical case. A family of Riemannian metrics is called monotone if its members are decreasing under stochastic mappings. These are the metrics to play the role of Fisher metric in the quantum case. Monotone metrics can be labeled by special operator monotone functions, according to Petz's Classification Theorem. The aim of this paper is to present an idea how one can narrow the set of monotone metrics from the statistical point of view, and to show that the monotone metrics which occur in the literature most often fit to this idea in qubit case.
\end{abstract}

1. Introduction. The idea in mathematical statistics that a statistical or informational distance between probability measures gives rise to a Riemannian metric is due to Rao [21] and was developed by Amari [1] and Streater [22] among others. The study of classical monotone metrics for parametric statistical manifolds was initiated by Cencov and Morozova [12]. They proved that only the Fisher information metric is monotone with respect to Markovian maps. In the quantum case Petz's Classification Theorem establishes a correspondence between monotone metrics and operator monotone functions. The main difference between classical and quantum information geometry is that in the classical case there is a unique (canonical) Riemannian metric on the space of classical finite probability distributions, but in the quantum case there are infinitely many such metric.

To narrow the infinite set of metrics in the quantum case we consider the statistical interpretations of the differential geometrical quantities. The volume of the geodesic ball with center state $D$ measures the statistical uncertainty. The first non trivial element in the series expansion of the volume of the geodesic ball is the scalar curvature, which

2000 Mathematics Subject Classification: Primary 53C20; Secondary 81Q99.

Key words and phrases: state space, monotone statistical metrics, scalar curvature.

Supported by OTKA T032662.

The paper is in final form and no version of it will be published elsewhere. 
measures the average statistical uncertainty of the state $D$. Therefore it is reasonable to believe that the other elements in this series expansion have similar statistical interpretation. Petz's conjecture is about the monotonicity of the scalar curvature with respect to the majorization relation when the state space is endowed with the Kubo-Mori metric. A monotone Riemannain metric is called $k$-admissible if the first $k$ element from the series expansion of the volume of the geodesic ball is monotone with respect to the majorization relation. Petz conjectured that the Kubo-Mori metric is 1-admissible. We present numerical simulations to show that the monotone metrics from the literature are at least 1, 2 and 3 -admissible in the qubit case.

2. Classical case. Probability distributions on a finite set $X_{n}=\{1, \ldots, n\}$ can be characterized by $(n-1)$ independent parameters $\left(p_{i}\right)_{i=1, \ldots, n-1}$ such that the density function $f$ is given as

$$
f\left(x, p_{1}, \ldots, p_{n}\right)=p_{i}, \text { if } x=i, \quad 1 \leq i \leq n,
$$

where $p_{1}+\cdots+p_{n}=1$. The open set of distributions on $X_{n}$ is

$$
\mathcal{P}_{n}=\left\{\left(p_{1}, \ldots, p_{n}\right) \mid 0<p_{i}<1, \sum_{i=1}^{n} p_{i}=1\right\} \text {. }
$$

The distribution $a=\left(a_{1}, \ldots, a_{n}\right) \in \mathcal{P}_{n}$ is called majorised by the distribution $b=$ $\left(b_{1}, \ldots, b_{n}\right) \in \mathcal{P}_{n}$, denoted by $a \prec b$, if the following inequalities hold for their decreasingly ordered set of parameters $\left(a_{i}^{(\downarrow)}\right)_{i=1, \ldots, n}$ and $\left(b_{i}^{(\downarrow)}\right)_{i=1, \ldots, n}$

$$
\sum_{l=1}^{k} a_{l}^{(\downarrow)} \leq \sum_{l=1}^{k} b_{l}^{(\downarrow)}
$$

for all $1 \leq k<n$. The intuitive meaning of the majorisation relation $a \prec b$ is that the distribution $a$ is more mixed or more chaotic than the distribution $b$.

The space $\mathcal{P}_{n}$ is an $(n-1)$ dimensional differentiable manifold. At a point $p=$ $\left(p_{1}, \ldots, p_{n}\right) \in \mathcal{P}_{n}$ the tangent space $\mathrm{T}_{p} \mathcal{P}_{n}$ can be identified with the vector space $\left\{\left(v_{1}, \ldots, v_{n}\right) \in \mathbb{R}^{n} \mid \sum_{i=1}^{n} v_{i}=0\right\}$. One can endow the space $\mathcal{P}_{n}$ with a Riemannian metric using one of the following technics.

1. The entropy of the distribution $p=\left(p_{1}, \ldots, p_{n}\right)$ is

$$
S(p)=-\sum_{i=1}^{n} p_{i} \log p_{i}
$$

This function is strictly concave on $\mathcal{P}_{n}$, therefore $(-1)$ times its Hessian is a Riemannian metric. Let us denote this metric by $g^{(1)}$.

2. The space $\mathcal{P}_{n}$ can be viewed as a part of the $(n-1)$ sphere $\mathbb{S}^{n-1}$ with the diffeomorphism

$$
\alpha: \mathcal{P}_{n} \rightarrow \mathbb{S}^{n-1}, \quad\left(p_{1}, \ldots, p_{n}\right) \mapsto\left(\sqrt{p_{1}}, \ldots, \sqrt{p_{n}}\right) .
$$

One can pull back the natural Riemannian metric of the sphere $\mathbb{S}^{n-1}$ with $\alpha$ to generate a Riemannian metric on the space of distributions $g^{(2)}$. 
3. Assume that $X$ and $\Xi$ are open connected subsets of $\mathbb{R}^{m}$ and $\mathbb{R}^{n}$ and for a function $f: X \times \Xi \rightarrow \mathbb{R}$ for every point $\xi \in \Xi$ the measure $f(\cdot, \xi) \mu$ is a probability measure on $X$, where $\mu$ is the Lebesgue measure on $\mathbb{R}^{m}$. If $f$ is smooth enough then the Fisher information matrix of the parametric distribution $f$ at a point $\xi=\left(\xi_{1}, \ldots, \xi_{n}\right) \in \Xi$ is

$$
[g(\xi)]_{i j}:=\int_{X} \frac{1}{f(x, \xi)} \frac{\partial f(x, \xi)}{\partial \xi_{i}} \frac{\partial f(x, \xi)}{\partial \xi_{i}} \mathrm{~d} \mu(x), \quad 1 \leq i, j \leq n .
$$

The Fisher information matrix of the statistical model $\mathcal{P}_{n}$ is an $(n-1) \times(n-1)$ matrix and depends on $(n-1)$ parameters, because $\operatorname{dim} \mathcal{P}_{n}=(n-1)$. This matrix at a point $p=\left(p_{1}, \ldots, p_{n}\right) \in \mathcal{P}_{n}$ is

$$
[g(p)]_{i j}:=\frac{1}{p_{i}} \delta_{i j}+\frac{1}{1-\sum_{k=1}^{n-1} p_{k}}, \quad 1 \leq i, j \leq n-1 .
$$

The matrix $\left[g(p)_{i j}\right]_{i, j=1, \ldots, n-1}$ is positive definite for every $p \in \mathcal{P}_{n}$. Rao used this matrix as a Riemannian metric first in 1945 [21].

4. One can determine a unique Riemannian metric on the space $\mathcal{P}_{n}$ using the Cencov Theorem. The Theorem is based on the concept of Markovian kernels. For finite sets $X_{n}$ and $X_{m}$ the function

$$
\kappa: X_{m} \times X_{n} \rightarrow \mathbb{R}^{+}, \quad(i, j) \mapsto \kappa_{j i},
$$

is called a Markovian map if for every $i \in X_{n}$ the equality $\sum_{j=1}^{m} \kappa_{j i}=1$ holds. A Markovian map $\kappa: X_{m} \times X_{n} \rightarrow \mathbb{R}^{+}$generates a function between manifolds

$$
\tilde{\kappa}: \mathcal{P}_{n} \rightarrow \mathcal{P}_{m}, \quad\left(p_{1}, \ldots, p_{n}\right) \mapsto\left(\sum_{i=1}^{n} \kappa_{0 i} p_{i}, \ldots, \sum_{i=1}^{n} \kappa_{m i} p_{i}\right),
$$

and this function has a natural action on the tangent bundle $\kappa^{*}: T \mathcal{P}_{n} \rightarrow T \mathcal{P}_{m}$.

Theorem 2.1 (Čencov, $[5,12]$ ). Assume that for every $n \in \mathbb{N}$ the pair $\left(\mathcal{P}_{n}, g_{n}\right)$ is a Riemannian-manifold. Let us consider the family of these spaces $\left(\mathcal{P}_{n}, g_{n}\right)_{n \in \mathbb{N}}$. If for every Markovian kernel $\kappa: X_{n} \times X_{m} \rightarrow \mathbb{R}$ the following monotonicity property holds

$$
g_{\tilde{\kappa}(p)}\left(\kappa^{*}(X), \kappa^{*}(X)\right) \leq g_{p}(X, X) \quad \forall p \in \mathcal{P}_{n}, \forall X \in \mathrm{T}_{p} \mathcal{P}_{n},
$$

then the family of metrics $\left(g_{n}\right)_{n \in \mathbb{N}}$ is unique up to a positive real number.

5. Relative entropies are often considered as distance functions between probability distributions. There are many relative entropy function [14], for example: for distributions $a=\left(a_{1}, \ldots, a_{n}\right)$ and $b=\left(b_{1}, \ldots, b_{n}\right)$ in $\mathcal{P}_{n}$ the Kullback-Liebler relative entropy [10] is

$$
D_{\mathrm{KL}}(a, b)=\sum_{k=1}^{n} a_{k} \log \frac{a_{k}}{b_{k}}
$$

the $\chi^{2}$ relative entropy is

$$
D_{\chi^{2}}(a, b)=\frac{1}{2} \sum_{k=1}^{n} a_{k}\left(\left(\frac{b_{k}}{a_{k}}\right)^{2}-1\right)
$$

and the delta relative entropy [23] is

$$
D_{\Delta}(a, b)=\sum_{k=1}^{n} \frac{\left(a_{k}-b_{k}\right)^{2}}{a_{k}+b_{k}}
$$


For an arbitrary point $p \in \mathcal{P}_{n}$ the bilinear map

$$
g_{p}^{(D)}: \mathrm{T}_{p} \mathcal{P}_{n} \times \mathrm{T}_{p} \mathcal{P}_{n} \rightarrow \mathbb{R},\left.\quad(\underline{x}, \underline{y}) \mapsto \frac{\partial^{2}}{\partial t \partial s} D(p+t \underline{x}+s \underline{y}, p)\right|_{t, s=0},
$$

is positive definite. So, these relative entropies - among others - generate Riemannian metrics on the space $\mathcal{P}_{n}$. It turns out that the generated metrics are equal, let us denote any of them by $g^{(5)}$.

It seems that we have defined many Riemannian metrics on the space of the classical discrete distributions $\mathcal{P}_{n}$, but these metrics coincide (up to a constant factor) according to the following theorem.

TheOREM 2.2. For the above defined metrics the equalities $g^{(1)}=4 g^{(2)}=g^{(3)}=g^{(5)}$ hold and they are equal to $g^{(4)}$ up to a positive real multiplier.

Proof. The metrics $g^{(1)}, g^{(2)}$ and $g^{(5)}$ can be computed easily, which implies the first equalities in the Theorem. It is enough to check that the metric $g^{(1)}$ fulfills the conditions in Čncov Theorem 2.1, which is again a simple computation.

REMARK 1. The above mentioned theorem means that the geometry of the space $\left(\mathcal{P}_{n}, g\right)$ is well-known, because the metric $g^{(2)}$ is nothing else but the pull back of the natural Riemannian metric of the sphere, so the space $\left(\mathcal{P}_{n}, g\right)$ is just a submanifold of the $n$ dimensional euclidean sphere $\mathbb{S}^{n-1}$. Therefore, the main differential geometrical invariants for example curvatures, geodesics, distances, volumes, etc. can be computed easily.

3. Noncommutative case. The quantum mechanical Hilbert space formalism gives a mathematical description of particles with spin of $\frac{n-1}{2}$. Concentrating on the spin part of nonrelativistic particles one can build a proper mathematical model in an $n$ dimensional complex Hilbert space. This is the simplest physical realization of an $n$-level quantum system. The states of an $n$-level system are identified with the set of real or complex $n \times n$ self-adjoint positive semidefinite matrices with trace 1 . The states are the noncommutative generalizations of the classical statistical model $\mathcal{P}_{n}$, because if we restrict ourselves to the diagonal matrices we get back the space $\mathcal{P}_{n}$. The states form a closed convex set in the space of matrices and its interior, the set of all strictly positive self-adjoint matrices with trace 1 becomes naturally a differentiable manifold, let $\mathcal{M}_{n}^{+}$ denote the set of these states.

Some concepts of the classical probability theory can be extended to the noncommutative case. The majorisation relation is one of them. The state $D_{1} \in \mathcal{M}_{n}^{+}$is called majorised by the state $D_{2} \in \mathcal{M}_{n}^{+}$, denoted by $D_{1} \prec D_{2}$ if the following inequalities hold for their decreasingly ordered set of eigenvalues $\left(\lambda_{i}^{(\downarrow)}\right)_{i=1, \ldots, n}$ and $\left(\mu_{i}^{(\downarrow)}\right)_{i=1, \ldots, n}$

$$
\sum_{l=1}^{k} \lambda_{l}^{(\downarrow)} \leq \sum_{l=1}^{k} \mu_{l}^{(\downarrow)}
$$

for all $1 \leq k<n$.

The state space $\mathcal{M}_{n}^{+}$is a differentiable manifold, the dimension of the real and complex state space is $\frac{(n-1)(n+2)}{2}$ and $\left(n^{2}-1\right)$. At a point $D \in \mathcal{M}_{n}^{+}$the tangent space $\mathrm{T}_{D} \mathcal{M}_{n}^{+}$ 
can be identified with the vector space of real or complex self-adjoint traceless matrices. One can try to endow the space $\mathcal{M}_{n}^{+}$with Riemannian metric as in the classical case.

1. John von Neumann defined the entropy in this quantum case in 1927 [13]. The von Neumann entropy of the state $D \in \mathcal{M}_{n}^{+}$is

$$
S(D)=-\operatorname{Tr} D \log D .
$$

This function is strictly concave on $\mathcal{M}_{n}^{+}$, therefore $(-1)$ times its Hessian is a Riemannian metric, called the Kubo-Mori or Bogoljubov metric. Let us denote this metric by $g^{(\mathrm{KM})}$.

2. The space $\mathcal{M}_{n}^{+}$can be viewed as a part of the euclidean sphere $\mathbb{S}^{d}$ with the diffeomorphism

$$
\alpha: \mathcal{M}_{n}^{+} \rightarrow \mathbb{S}^{d} \quad D \mapsto \sqrt{D}
$$

where $d=\frac{(n-1)(n+2)}{2}$ for real state space and $d=\left(n^{2}-1\right)$ for complex state space. One can pull back the natural Riemannian metric of the sphere $\mathbb{S}^{d}$ with $\alpha$ to generate a Riemannian metric on the state space $g^{(\mathrm{WY})}$, which is called the Wigner-Yanase metric.

3. One runs into difficulties with the generalization of the Fisher information matrix. The main problem is that in the definition of the classical Fisher information (1) the quantities were real numbers (commutative quantities), but in this matrix setting the analog quantities become matrices (noncommutative quantities). It means that one can define many noncommutative Fisher type information quantities, for example using the generalized logarithmic derivatives which were introduced by Petz and Tóth [20].

4. The noncommutative extension of the Čencov Theorem was given by Petz in 1996. The stochastic maps are the counterpart of the Markovian maps in this setting. A linear map between matrix spaces $T: M_{n} \rightarrow M_{m}$ is called stochastic if it is trace preserving and completely positive.

Theorem 3.1 (Petz, [17]). Assume that for every $n \in \mathbb{N}$ the pair $\left(\mathcal{M}_{n}^{+}, K_{n}\right)$ is a Riemannian-manifold. Let us consider the family of these spaces $\left(\mathcal{M}_{n}^{+}, K_{n}\right)_{n \in \mathbb{N}}$. If for every stochastic map $T: M_{n} \rightarrow M_{m}$ the following monotonicity property holds

$$
K_{T(D)}(T(X), T(X)) \leq K_{D}(X, X) \quad \forall D \in \mathcal{M}_{n}^{+}, \forall X \in \mathrm{T}_{D} \mathcal{M}_{n}^{+}
$$

then there exists an operator monotone function $f: \mathbb{R}^{+} \rightarrow \mathbb{R}$ with the property $f(x)=$ $x f\left(x^{-1}\right)$, such that

$$
K_{D}(X, Y)=\operatorname{Tr}\left(X\left(R_{n, D}^{\frac{1}{2}} f\left(L_{n, D} R_{n, D}^{-1}\right) R_{n, D}^{\frac{1}{2}}\right)^{-1}(Y)\right),
$$

where $L_{n, D}$ and $R_{n, D}$ are defined as $L_{n, D}(X)=D X$ and $R_{n, D}(X)=X D$.

These metrics are considered as the noncommutative generalizations of the Fisherinformation. Because of the monotonicity property these metrics are called monotone metrics. One can use (3) to define a monotone metric on the state space starting from an appropriate operator monotone function.

5. The first relative entropy definition in the quantum setting is due to Umegaki [24]:

$$
S_{\mathrm{U}}\left(D_{1}, D_{2}\right)=\operatorname{Tr} D_{1}\left(\log D_{1}-\log D_{2}\right) .
$$

Petz based the generalization of the classical relative entropy on relative modular operators $[14,18,19]$. For an operator convex function $g:] 0, \infty[\rightarrow \mathbb{R}$ with property $g(1)=0$ 
the function

$$
S_{g}(\cdot, \cdot): \mathcal{M}_{n}^{+} \times \mathcal{M}_{n}^{+} \rightarrow \mathbb{R} \quad\left(D_{1}, D_{2}\right) \mapsto S_{g}\left(D_{1}, D_{2}\right)=\operatorname{Tr}\left(D_{1}^{1 / 2} g\left(L_{D_{2}} R_{D_{1}}^{-1}\right) D_{1}^{1 / 2}\right)
$$

is called the $g$-relative entropy.

The operator convex function $g(x)=\frac{(x-1) \log x}{2}$ generates the Umegaki relative entropy, $g(x)=\frac{(x-1)^{2}}{2}$ generates the quadratic relative entropy

$$
S_{\mathrm{Q}}\left(D_{1}, D_{2}\right)=\frac{1}{2} \operatorname{Tr}\left(D_{1}-D_{2}\right) D_{1}^{-1}\left(D_{1}-D_{2}\right)
$$

and $g(x)=\frac{(x-1)^{2}}{1+x}$ generates the Bures relative entropy

$$
S_{\mathrm{B}}\left(D_{1}, D_{2}\right)=\operatorname{Tr}\left(D_{1}-D_{2}\right)\left(L_{D_{2}}+R_{D_{1}}\right)^{-1}\left(D_{1}-D_{2}\right) .
$$

On the space of diagonal matrices these relative entropies are the classical ones, that is $S_{\mathrm{U}}=D_{\mathrm{KL}}, S_{\mathrm{Q}}=D_{\chi^{2}}$ and $S_{\mathrm{B}}=D_{\Delta}$. These relative entropies generate Riemannian metrics on the state space $\mathcal{M}_{n}^{+}$, as in the classical case, because for an arbitrary state $D \in \mathcal{M}_{n}^{+}$the bilinear map

$$
g_{D}^{(S)}: \mathrm{T}_{D} \mathcal{M}_{n}^{+} \times\left.\mathrm{T}_{D} \mathcal{M}_{n}^{+} \rightarrow \mathbb{R} \quad(X, Y) \mapsto \frac{\partial^{2}}{\partial t \partial s} S(D+t X+s Y, D)\right|_{t, s=0}
$$

is positive definite. Let us denote the generated metrics by $g^{(\mathrm{U})}, g^{(\mathrm{Q})}$ and $g^{(\mathrm{B})}$. The metrics $g^{(\mathrm{Q})}$ and $g^{(\mathrm{B})}$ are called greatest and smallest monotone metrics.

These methods gave the same Riemannian metric in the classical case, but in this noncommutative setting we have the following Theorem.

THEOREM 3.2. The metrics $g^{(\mathrm{KM})}$ and $g^{(\mathrm{WY})}$ are monotone metrics and the corresponding operator monotone functions are $\frac{x-1}{\log x}$ and $\frac{(\sqrt{x}-1)^{2}}{4}$. The metric generated by the Umegaki relative entropy is equal to the Kubo-Mori metric, that is $g^{(\mathrm{U})}=g^{(\mathrm{KM})}$. The greatest and smallest monotone metrics are monotone metrics, with operator monotone functions $\frac{2 x}{1+x}$ and $\frac{1+x}{2}$, respectively.

REMARK 2. After these steps we conclude that there is no unique extension of the classical Fisher information, and the most general Fisher information is given by the Petz formula (3). It means that the canonical geometry of the quantum mechanical state space can be labeled by special operator monotone functions. So the differential geometrical quantities of the state space depend on the operator monotone function which generates its Riemannian metric.

4. Series expansion of the volume. Let $(M, g)$ be an $n$-dimensional Riemannian geometry. The geodesic ball with center $p \in M$ and radius $R$ is defined to be

$$
B_{R}(p):=\{x \in M: \operatorname{dist}(p, x)<R\} .
$$

For a fixed $p$ point let $V_{R}(p)$ denote the volume of $B_{R}(p)$. For flat manifolds $V_{R}(p)$ is equal to the volume of the euclidean ball, but in general spaces $V_{R}(p)$ can be greater or smaller, because of the curvature. The first series expansion of $V_{R}(p)$ was given by 
Bertrand, Diguet and Puiseux in 1848 [4]. They published the formula

$$
V_{R}(p)=\pi R^{2}\left[1-\frac{K(p)}{12} R^{2}+O\left(R^{4}\right)\right]
$$

for surfaces in $\mathbb{R}^{3}$, where $K(p)$ denotes the Gaussian curvature at a point $p$. This result was generalized for arbitrary Riemannian manifolds by Vermeil in 1917 [25] and by Hotelling in 1939 [9]. The next element from the series expansion was given by Gray in 1973 [7] and another element was given by Gray and Vanhecke in 1979 [8].

Assume that $(M, g)$ is a Riemannian manifold. At a given point $p \in M$ the metric tensor is a matrix $\left[g(p)_{i k}\right]_{i, k=1, \ldots, \operatorname{dim} M}$, the inverse of this matrix is denoted by $\left[g(p)^{i k}\right]_{i, k=1, \ldots, \operatorname{dim} M}$. From this point we will use the Einstein summation rule, that is: if an index occurs once as a lower index and once as an upper index we have to sum up over that index from 1 to $\operatorname{dim} M$. To understand and use the series expansion of the volume first we introduce the most basic differential geometrical quantities (the Christoffel symbol, the Riemannian curvature tensor, the Ricci tensor and the scalar curvature)

$$
\begin{aligned}
& \Gamma_{i j}^{. . m}=g^{k m} \frac{1}{2}\left(\partial_{i} g_{j k}+\partial_{j} g_{i k}-\partial_{k} g_{i j}\right), \\
& R_{i j k}^{. . l}=\partial_{i} \Gamma_{j k}^{. . l}-\partial_{j} \Gamma_{i k}^{. l l}+\Gamma_{j k}^{. . m} \Gamma_{i m}^{. l}-\Gamma_{i k}^{. . m} \Gamma_{j m}^{. l}, \\
& \operatorname{Ric}_{j k}=R_{i j k}^{\ldots i} \\
& \text { Scal }=\operatorname{Ric}_{i j} g^{i j} \text {. }
\end{aligned}
$$

From these basic quantities we define some versions of them

$$
\begin{aligned}
& \operatorname{Ric}^{k l}=\operatorname{Ric}_{i j} g^{i k} g^{j l}, \quad \operatorname{Ric}_{i}^{j}=\operatorname{Ric}_{i k} g^{k j}, \\
& R^{i j k l}=R_{\text {mnop }} g^{m i} g^{n j} g^{o k} g^{p l}, \\
& R_{j k l}^{i}=R_{n j k l} g^{n i}, \\
& R_{i j}^{. . k l}=R_{i j m n} g^{m k} g^{n l}, \\
& R_{. k . l}^{i . j}=R_{m k n l} g^{m i} g^{n j},
\end{aligned}
$$

and some new summations of them

$$
\begin{array}{lll}
\| \operatorname{Ric}^{2}=\operatorname{Ric}_{i j} \operatorname{Ric}^{i j}, & \|R\|^{2}=R_{i j k l} R^{i j k l}, & \check{\operatorname{Ric}}=\operatorname{Ric}_{i}^{. j} \operatorname{Ric}_{j}^{. k} \operatorname{Ric}_{k}^{. i}, \\
\langle\operatorname{Ric}, \dot{R}\rangle=\operatorname{Ric}_{i j} R^{i p q r} R_{. p q r}^{j}, & \langle\operatorname{Ric} \otimes \operatorname{Ric}, \tilde{R}\rangle=\operatorname{Ric}^{i j} \operatorname{Ric}^{k l} R_{i k j l}, & \check{R}=R_{i j}^{. . k l} R_{k l}^{. . p q} R_{p q}^{. i j}, \\
\bar{R}=R_{i k j l} R_{. p . q}^{k . l} R^{p i q j} & &
\end{array}
$$

and finally some derivatives of them

$$
\begin{aligned}
& \| \nabla \text { Scal } \|^{2}=\left(\partial_{i} \operatorname{Scal}\right)\left(\partial_{j} \operatorname{Scal}\right) g^{i j} \\
& (\nabla \text { Ric })_{i j k}=\partial_{k} \operatorname{Ric}_{i j}-\Gamma_{i k}^{. . p} \operatorname{Ric}_{p j}-\Gamma_{j k}^{. . p} \operatorname{Ric}_{i p}, \\
& \| \nabla \text { Ric } \|^{2}=(\nabla \operatorname{Ric})_{i j k}(\nabla \text { Ric })_{l m n} g^{l i} g^{m j} g^{n k}, \\
& \alpha(\operatorname{Ric})=(\nabla \text { Ric })_{j k i}(\nabla \text { Ric })_{l m n} g^{l i} g^{m j} g^{n k}, \\
& (\nabla R)_{i j k l m}=\partial_{m} R_{i j k l}-\Gamma_{i m}^{. . p} R_{p j k l}-\Gamma_{j m}^{. . p} R_{i p k l}-\Gamma_{k m}^{. . p} R_{i j p l}-\Gamma_{l m}^{. . p} R_{i j k m},
\end{aligned}
$$




$$
\begin{gathered}
\|\nabla R\|^{2}=(\nabla R)_{i j k l m}(\nabla R)_{n o p q r} g^{n i} g^{o j} g^{p k} g^{q l} g^{r m} \\
\Delta \mathrm{Scal}=\left(\partial_{i} \partial_{j} \mathrm{Scal}\right) g^{i j}-\Gamma_{i j}^{. . p}\left(\partial_{p} \mathrm{Scal}\right) g^{i j} \\
\langle\Delta \mathrm{Ric}, \mathrm{Ric}\rangle=\left(\partial_{l}(\nabla \mathrm{Ric})_{i j k}\right) g^{k l} \operatorname{Ric}^{i j}-\Gamma_{i l}^{. . p}(\nabla \mathrm{Ric})_{p j k} g^{k l} \mathrm{Ric}^{i j} \\
-\Gamma_{j l}^{. . p}(\nabla \mathrm{Ric})_{i p k} g^{k l} \operatorname{Ric}^{i j}-\Gamma_{k l}^{. . p}(\nabla \mathrm{Ric})_{i j p} g^{k l} \mathrm{Ric}^{i j}, \\
\left\langle\nabla^{2} \mathrm{Scal}, \mathrm{Ric}\right\rangle=\left(\partial_{j} \partial_{i} \mathrm{Scal}\right) \operatorname{Ric}^{i j}-\Gamma_{i j}^{. . p}\left(\partial_{p} \mathrm{Scal}\right) \mathrm{Ric}^{i j} \\
\langle\Delta R, R\rangle=R^{i j k l} g^{m n}\left(\partial_{n}(\nabla R)_{i j k l m}\right)-\Gamma_{i n}^{. . p}(\nabla R)_{p j k l m} R^{i j k l} g^{m n} \\
-\Gamma_{j n}^{. . p}(\nabla R)_{i p k l m} R^{i j k l} g^{m n}-\Gamma_{k n}^{. . p}(\nabla R)_{i j p l m} R^{i j k l} g^{m n} \\
-\Gamma_{l n}^{. . p}(\nabla R)_{i j k p m} R^{i j k l} g^{m n}-\Gamma_{m n}^{. . p}(\nabla R)_{i j k l p} R^{i j k l} g^{m n}, \\
\Delta^{2} \mathrm{Scal}=\left(\partial_{i} \partial_{j}(\Delta \mathrm{Scal})\right) g^{i j}-\Gamma_{i j}^{. . p}\left(\partial_{p}(\Delta \mathrm{Scal})\right) g^{i j} .
\end{gathered}
$$

Theorem 4.1 (Gray and Vanhecke, $[8]$ ). Let $(M, g)$ be an $n$ dimensional Riemannian manifold. For an arbitrary point $p \in M$ the series expansion of the volume of the geodesic ball $V_{R}(p)$ (with center $p$ and radius $R$ ) is the following:

$$
\begin{aligned}
& V_{R}(p)=\frac{R^{n} \pi^{n / 2}}{\Gamma\left(\frac{n}{2}+1\right)}\left[1-\frac{\mathrm{Scal}}{6(n+2)} R^{2}+\frac{-3\|R\|^{2}+8\|\mathrm{Ric}\|^{2}-5 \mathrm{Scal}^{2}-18(\Delta \mathrm{Scal})}{360(n+2)(n+4)} R^{4}\right. \\
& +\frac{1}{720(n+2)(n+4)(n+6)}\left(-\frac{5}{9} \mathrm{Scal}^{3}-\frac{8}{3} \mathrm{Scal}\|\mathrm{Ric}\|^{2}+\mathrm{Scal}\|R\|^{2}+\frac{64}{63} \mathrm{Ric}\right. \\
& +\frac{64}{21}\langle\operatorname{Ric} \otimes \operatorname{Ric}, \tilde{R}\rangle+\frac{32}{7}\langle\operatorname{Ric}, \dot{R}\rangle+\frac{110}{63} \check{R}+\frac{200}{63} \bar{R}+\frac{45}{7}\|\nabla \mathrm{Scal}\|^{2}+\frac{45}{14}\|\nabla \mathrm{Ric}\|^{2} \\
& +\frac{45}{7} \alpha(\operatorname{Ric})-\frac{45}{14}\|\nabla R\|^{2}+6 \mathrm{Scal}(\Delta \mathrm{Scal})+\frac{48}{7}\langle\Delta \mathrm{Ric}, \mathrm{Ric}\rangle+\frac{54}{7}\left\langle\nabla^{2} \text { Scal, Ric }\right\rangle \\
& \left.\left.\quad-\frac{30}{7}\langle\Delta R, R\rangle-\frac{45}{7}\left(\Delta^{2} \mathrm{Scal}\right)\right) R^{6}+O\left(R^{8}\right)\right] .
\end{aligned}
$$

REMARK 3. For example one curvature invariant which occurs in the series expansion is

$$
\|\nabla R\|^{2}=\sum_{i, j, k, l, m, o, p, q, r, s=1}^{n}(\nabla R)_{i j k l m}(\nabla R)_{o p q r s} g^{i o} g^{j p} g^{k q} g^{l r} g^{m s},
$$

so to compute the higher order curvature invariants in a concrete application is nearly hopeless, even in low dimensions, because it requires to sum up $n^{10}$ elements.

In the classical case the series expansion of the geodesic ball is given by the following theorem. It is worth remarking that the series expansion in this case is independent of the center of the ball.

Theorem 4.2 (Classical case). For the Riemannian geometry $\left(\mathcal{P}_{n}, g\right)$ the series expansion of the volume of the geodesic ball is 


$$
\begin{aligned}
V_{n}(R)=\frac{r^{n-1} \pi^{(n-1) / 2}}{\Gamma\left(\frac{n-1}{2}+1\right)}[ & 1-\frac{(n-1)(n-2)}{24(n+1)} R^{2}++\frac{(n-1)(n-2)(5 n-12)}{5760(n+3)} R^{4} \\
& \left.-\frac{(n-1)(n-2)\left(35 n^{2}-182 n+240\right)}{2903040(n+5)} R^{6}+O\left(R^{8}\right)\right] .
\end{aligned}
$$

Proof. Let us use

$$
\varphi: \mathcal{P}_{n} \rightarrow \mathbb{R}^{n-1}, \quad\left(p_{1}, \ldots, p_{n}\right) \mapsto\left(p_{1}, \ldots, p_{n-1}\right),
$$

the global coordinate system on the manifold $\mathcal{P}_{n}$. The components of the metric tensor $\left[g_{i j}\right]_{i, j=1, \ldots, n-1}$ is given by (2). The inverse of the metric tensor is $g^{i j}=-p_{i} p_{j}+\delta_{i j} p_{i}$. The Christoffel symbols, Ricci tensor and scalar curvature are

$$
\begin{aligned}
& \Gamma_{i j}^{. . k}=\frac{1}{2}\left(\frac{p_{k}}{1-\sum_{j=1}^{n-1} p_{j}}+\delta_{i j} \frac{p_{k}}{p_{i}}-\delta_{i j} \delta_{j k} \frac{1}{p_{j}}\right), \\
& \operatorname{Ric}_{j k}=\frac{n-2}{4}\left(\frac{1}{1-\sum_{i=1}^{n-1} p_{i}}+\delta_{j k} \frac{1}{p_{j}}\right) \\
& \text { Scal }=\frac{(n-1)(n-2)}{4} .
\end{aligned}
$$

The other derived quantities are the following.

$$
\begin{array}{lll}
\operatorname{Ric}^{i j}=\frac{n-2}{4}\left(-p_{i} p_{j}+\delta_{i j} p_{i}\right), & \|\operatorname{Ric}\|^{2}=\frac{(n-1)(n-2)^{2}}{16}, & \Delta \text { Scal }=0, \\
\|R\|^{2}=\frac{(n-1)(n-2)}{8}, & \check{\operatorname{Ric}}=\frac{(n-1)(n-2)^{3}}{64}, & \| \nabla \text { Ric } \|^{2}=0, \\
\langle\Delta \operatorname{Ric}, \operatorname{Ric}\rangle=0, & \| \nabla \text { Scal } \|^{2}=0, & \left\langle\nabla^{2} \text { Scal, Ric }\right\rangle=0, \\
\langle\operatorname{Ric} \otimes \operatorname{Ric}, \tilde{R}\rangle=-\frac{(n-1)(n-2)^{3}}{64}, & \alpha(\operatorname{Ric})=0, & \nabla^{2} \text { Scal }=0, \\
\langle\operatorname{Ric}, \dot{R}\rangle=\frac{(n-1)(n-2)^{2}}{32}, & \check{R}=-\frac{(n-1)(n-2)}{16}, & \|\nabla R\|^{2}=0, \\
\bar{R}=-\frac{(n-1)(n-2)(n-3)}{64}, & \langle\Delta R, R\rangle=0 .
\end{array}
$$

Substituting these quantities into the series expansion of the volume one concludes the proof of the Theorem.

The volume of the geodesic ball with center $p \in M$ can be written in the form

$$
V_{R}(p)=C_{n} R^{n}\left(1+\sum_{i=1}^{\infty} \alpha_{i}(p) R^{2 i}\right),
$$

where only the functions $\left(\alpha_{i}\right)_{i \in \mathbb{N}}$ depend on the point $p$. Gray and Vanhecke computed the functions $\alpha_{1}, \alpha_{2}$ and $\alpha_{3}$.

We will focus on the geometry of state space (quantum information geometry). It is widely believed that in the above mentioned information geometrical setting (both classical and quantum case) the quantity $V_{R}(p)$ has statistical interpretation. Therefore the functions $\left(\alpha_{i}\right)_{i \in \mathbb{N}}$ in the series expansion also can interpreted in the language of statistics. For example the first nontrivial function $\alpha_{1}$ corresponds to the scalar curvature, which measures the average statistical uncertainty as shown by Petz [15]. It is reasonable 
to expect that more mixed states are less distinguishable than less mixed states. It means mathematically that in this case the scalar curvature of a Riemann structure should have the following monotonicity property: if $D_{1}$ is more mixed than $D_{2}$ then $\operatorname{Scal}\left(D_{2}\right)$ should be smaller then $\operatorname{Scal}\left(D_{1}\right)$. This is known as the Petz conjecture, if the state space is endowed with the Kubo-Mori metric [16]. Petz proved the conjecture for $2 \times 2$ complex density matrices [16]. The scalar curvature of the state endowed with the Kubo-Mori metric was given by Michor, Petz and Andai for the real state space [11] and by Dittmann for the complex state space [6]. Partial results about the conjecture can be found in [3]. Taking into account the statistical interpretation of the volume and the first nontrivial element in the series expansion we conclude that those monotone metrics are important in some sense from statistical point of view, for which the functions $\left(\alpha_{i}\right)_{i \in \mathbb{N}}$ are monotone with respect to the majorisation relation. This is the concept behind the following definition.

Definition 1. The monotone Riemannian metric $g$ on the state space $\mathcal{M}_{n}^{+}$is called $k$-admissible if for any states $D_{1}, D_{2} \in \mathcal{M}_{n}^{+}$the implication

$$
D_{1} \prec D_{2} \Longrightarrow \forall i \in\{1, \ldots, k\}: \alpha_{i}\left(D_{1}\right) \leq \alpha_{i}\left(D_{2}\right)
$$

holds, and $g$ is admissible if it is $k$-admissible for every $k \in \mathbb{N}$.

In this sense Petz conjecture means that the Kubo-Mori metric is 1-admissible. Because of the central role of the entropy in the theory of (quantum) statistical mechanics we conjecture that the metric generated by the entropy is admissible.

Conjecture 4.1 (Generalized Petz conjecture). The Kubo-Mori metric is admissible.

From a given operator monotone function, which generates monotone metric, it is not easy to decide whether the generated metric is admissible or not, because the functions $\alpha_{i}$ are rather complicated.

5. Qubit case. In the simplest quantum case, dealing with $2 \times 2$ matrices we can use the Stokes parametrization, that is every state $D$ can be uniquely written in the form

$$
D=\frac{1}{2}\left(I+x_{1} \sigma_{1}+x_{2} \sigma_{2}+x_{3} \sigma_{3}\right)
$$

where $\left(\sigma_{i}\right)_{i=1,2,3}$ are the Pauli matrices and $\left(x_{1}, x_{2}, x_{3}\right) \in \mathbb{R}^{3}$ with $x_{1}^{2}+x_{2}^{2}+x_{3}^{2} \leq 1$. The interior of the set of states can be identified with the open unit ball in $\mathbb{R}^{3}$ by this parametrization. For a fixed state $D \in \mathcal{M}_{2}^{+}$(qubit) from the open unit ball denote by $r$ the distance between $D$ and the origin. If $\lambda_{1}$ and $\lambda_{2}$ are the eigenvalues of $D$ then $r=\left|\lambda_{1}-\lambda_{2}\right|$, and this $r$ measures the purity of the state: $r=0$ corresponds to the most mixed state and in a pure state $r=1$.

TheOREM 5.1. Let $D \in \mathcal{M}_{2}^{+}$and $r=2 \lambda_{1}-1$ where $\lambda_{1}$ is an eigenvalue of $D$ and assume that the monotone metric of $\mathcal{M}_{2}^{+}$comes from a function $f$. Then the function $\alpha_{1}$ at $D$ is

$$
\begin{gathered}
\alpha_{1}(r)=-\frac{7(r-1)\left[f^{\prime}\left(\frac{1-r}{1+r}\right)\right]^{2}}{15(1+r)^{3}\left[f\left(\frac{1-r}{1+r}\right)\right]^{2}}-\frac{\left(r^{2}+7 r-6\right) f^{\prime}\left(\frac{1-r}{1+r}\right)}{15(1+r)^{2} r f\left(\frac{1-r}{1+r}\right)}-\frac{4(1-r) f^{\prime \prime}\left(\frac{1-r}{1+r}\right)}{15(1+r)^{3} f\left(\frac{1-r}{1+r}\right)} \\
-\frac{(1+r) f\left(\frac{1-r}{1+r}\right)}{15 r^{2}}-\frac{3 r^{3}+5 r^{2}+8 r-4}{60(1+r) r^{2}} .
\end{gathered}
$$


Proof. The scalar curvature of $\mathcal{M}_{2}^{+}$at a state $D \in \mathcal{M}_{2}^{+}$was expressed in [2] in terms of the function $f$. To prove this Theorem it is enough to substitute that expression into the series expansion of the volume.

One can show that the volume of the geodesic ball with a given center $D \in \mathcal{M}_{2}^{+}$ and with radius $R$ depends only on the parameters $r$ (which measures the purity of the state $D)$ and $R$, we will denote this volume by $V_{R}(r)$. The quantity $V_{R}(r)$ for a general monotone Riemannian metric cannot be expressed explicitly, except when the center of the ball is the most mixed state.

Theorem $5.2([2])$. Assume that the monotone metric of $\mathcal{M}_{2}^{+}$is generated by $f$. The volume of the geodesic ball with radius $R$ around the most mixed state (when $r=0)$ is

$$
V_{R}(0)=4 \pi \int_{0}^{R} \frac{t^{2}}{(1+t) \sqrt{1-t^{2}} f\left(\frac{1-t}{1+t}\right)} \mathrm{d} t .
$$

The series expansion of this volume is

$$
\begin{aligned}
V_{R}(0)=\frac{4 \pi R^{3}}{3}[ & 1-\frac{1+6 f^{\prime \prime}(1)}{5} R^{2}+\frac{2-30 f^{(4)}(1)+150 f^{\prime \prime}(1)+180\left(f^{\prime \prime}(1)\right)^{2}}{105} R^{4} \\
& +\left(-\frac{1}{945}+\frac{14}{9} f^{(4)}(1)+\frac{178}{15} f^{\prime \prime}(1)-4\left(f^{\prime \prime}(1)\right)^{2}-\frac{4}{135} f^{(6)}(1)\right. \\
& \left.\left.+\frac{32}{3} f^{(3)}(1)+\frac{8}{9} f^{\prime \prime}(1) f^{(4)}(1)-\frac{8}{3}\left(f^{\prime \prime}(1)\right)^{3}\right) R^{6}+O\left(R^{8}\right)\right] .
\end{aligned}
$$

To express the functions $\alpha_{2}$ and $\alpha_{3}$ one has to continue the differential geometrical computations which was given in [2]. The results for general function $f$ will be quite complicated formulas. Using mathematical programs (Maple or Mathematica) one can compute and visualize the functions $\alpha_{2}$ and $\alpha_{3}$ in concrete examples.

EXAMPLE 1. The monotone metric generated by the function $f(x)=\frac{1+x}{2}$ is the smallest metric. The series expansion of the volume of a geodesic ball with center $D \in \mathcal{M}_{2}^{+}$is

$$
V_{R}(r)=\frac{4 \pi R^{3}}{3}\left(1-\frac{1}{5} R^{2}+\frac{2}{105} R^{4}-\frac{1}{945} R^{6}+O\left(R^{8}\right)\right) .
$$

The functions $\left(\alpha_{i}\right)_{i=1,2,3}$ do not depend on the center state $D$, as in the classical case. This is the only monotone metric with this property known to the author.

EXAMPLE 2. The monotone metric generated by the function $f(x)=\frac{2 x}{1+x}$ is the largest metric. The series expansion of $V_{R}(r)$ is

$$
\begin{aligned}
V_{R}(r) & =\frac{4 \pi R^{3}}{3}\left(1-\frac{1}{15} \frac{6-r^{2}}{1-r^{2}} R^{2}++\frac{1}{1575} \frac{3 r^{4}+50 r^{2}+225}{\left(1-r^{2}\right)^{2}} R^{4}\right. \\
& \left.+\frac{1}{33075} \frac{r^{6}-84 r^{4}-2380 r^{2}-2170}{\left(1-r^{2}\right)^{3}} R^{6}+O\left(R^{8}\right)\right) .
\end{aligned}
$$

The functions $\alpha_{1}, \alpha_{2}$ and $\alpha_{3}$ are plotted with solid, dashed and dotted lines in the following picture. 


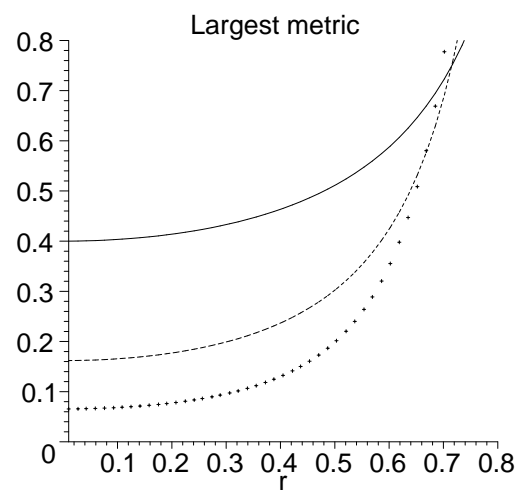

Example 3. The Kubo-Mori metric is generated by the function $f(x)=\frac{x-1}{\log x}$. The volume expansion for a fixed $D \in \mathcal{M}_{2}^{+}$qubit can be computed, but the result is a rather complicated formula. The picture shows the behavior of the functions $\left(\alpha_{i}\right)_{i=1,2,3}$.

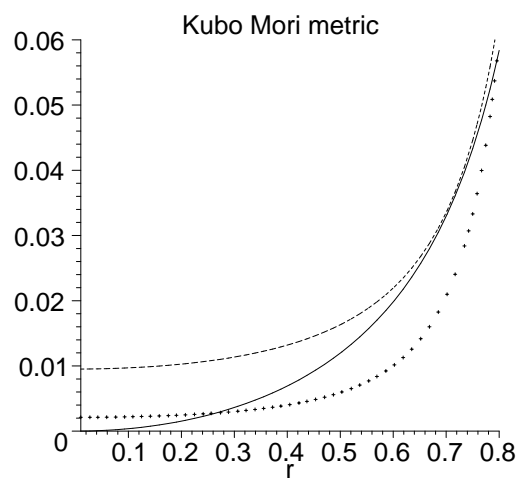

EXAMPLE 4. The function $f(x)=\frac{2 x^{p+1 / 2}}{1+x^{2 p}}$ generates a monotone metric for every parameter $p \in\left[0, \frac{1}{2}\right]$. In this case the functions $\left(\alpha_{i}\right)_{i=1,2,3}$ depend not only $r$, but on the parameter of the metric $p$ as well. In this parametric case $\alpha_{i}$ are rather complicated expressions, for example the function $\alpha_{3}$ consists of more than 500 summands. The function $\alpha_{1}$ is the brightest and $\alpha_{3}$ is the darkest in the next picture.
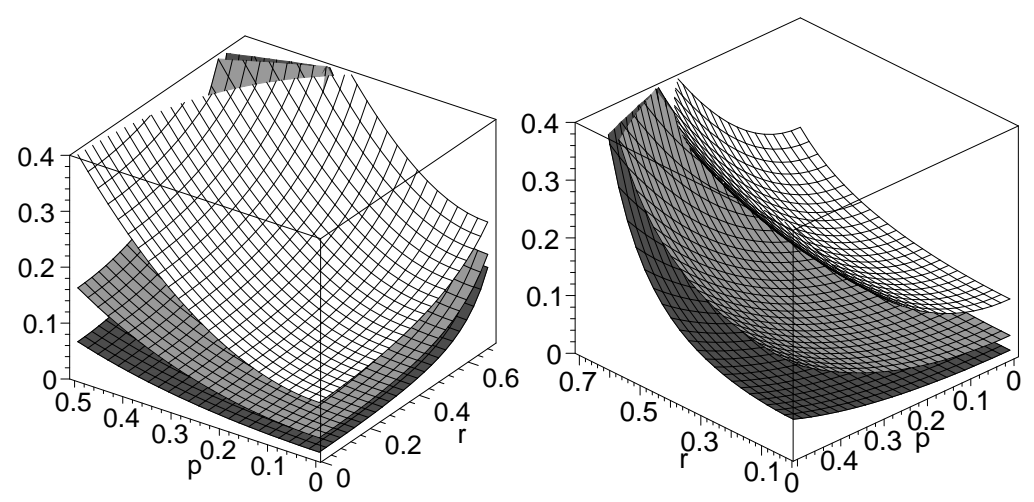
From the above mentioned metrics numerical simulations show that they are 3admissible. (Every function $\left(\alpha_{i}\right)_{i=1,2,3}$ is increasing with respect to the parameter $r$.) The same property can be proved numerically about the monotone metric generated by the function $f_{\beta}(x)=\frac{\beta(1-\beta)(x-1)^{2}}{\left(x^{\beta}-1\right)\left(x^{1-\beta}-1\right)}$, where $0<\beta<1$. But this is not true for every monotone metric even in the qubit case.

EXAMPLE 5. The next picture shows the strange behavior of the functions $\left(\alpha_{i}\right)_{i=1,2,3}$ if the monotone metric is generated by the function $f(x)=\frac{9 x^{2}+82 x+9}{5(x+1)}$.

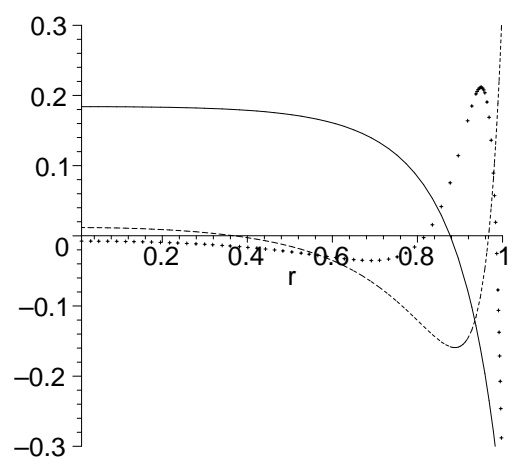

6. Conclusion. We showed numerically that the well known monotone metrics are at least 3 -admissible in the qubit case. (We conjecture that they are admissible.) For $n \times n$ density matrices the computing time of the quantities $\left(\alpha_{i}\right)_{i=1,2,3}$ is about $n^{20}$. To compute these quantities on the space of $3 \times 3$ complex density matrices requires about 3000 times longer computing time, so it remains an open question whether these metrics are admissible or not in the space of higher level quantum systems.

\section{References}

[1] S. Amari, Differential-Geometrical Methods in Statistics, Lecture Notes in Statistics 28, Springer-Verlag, New York, 1985.

[2] A. Andai, Monotone Riemannian metrics on density matrices with non-monotone scalar curvature, J. Math. Phys. 44 (2003), 3675-3688.

[3] A. Andai, On the monotonicity conjecture for the curvature of the Kubo-Mori metric, arXiv:math-ph/0310064.

[4] J. Bertrand, C. F. Diguet and V. Puiseux, Démonstration d'un théorème de Gauss, Journal de Mathématiques 13 (1848), 80-90.

[5] N. N. Čencov, Statistical Decision Rules and Optimal Inference, Translations of Mathematical Monographs 53, American Mathematical Society, Providence, R.I., 1982.

[6] J. Dittmann, On the curvature of monotone metrics and a conjecture concerning the Kubo-Mori metric, Linear Algebra Appl. 315 (2000), 93-112.

[7] A. Gray, The volume of a small geodesic ball of a Riemannian manifold, Michigan Math. J. 20 (1973), 329-344.

[8] A. Gray and L. Vanhecke, Riemannian geometry as determined by the volumes of small geodesic balls, Acta Math. 142 (1979), 157-198. 
[9] H. Hotteling, Tubes and spheres in n-spaces, and a class of statistical problems, Amer. J. Math. 61 (1939), 440-460.

[10] S. Kullback and R. A. Leibler, On information and sufficiency, Ann. Math. Statistics 22 (1951), 79-86.

[11] P. W. Michor, D. Petz and A. Andai, On the curvature of a certain Riemannian space of matrices, Infin. Dimens. Anal. Quantum Probab. Relat. Top. 3 (2000), 199-212.

[12] E. A. Morozova and N. N. Cencov, Markov invariant geometry on state manifolds, in: Current Problems in Mathematics. Newest Results (Russian), Akad. Nauk SSSR Vsesoyuz. Inst. Nauchn. i Tekhn. Inform. 36, Moscow, 1989, 69-102.

[13] J. von Neumann, Thermodynamik quantenmechanischer Gesamtheiten, Gött. Nachr. (1927), 273-291.

[14] M. Ohya and D. Petz, Quantum Entropy and its Use, Texts and Monographs in Physics, Springer-Verlag, Berlin, 1993.

[15] D. Petz, Covariance and Fisher information in quantum mechanics, J. Phys. A 35 (2002), 929-939.

[16] D. Petz, Geometry of canonical correlation on the state space of a quantum system, J. Math. Phys. 35 (1994), 780-795.

[17] D. Petz, Monotone metrics on matrix spaces, Linear Algebra Appl. 244 (1996), 81-96.

[18] D. Petz, Quasi-entropies for finite quantum systems, Rep. Math. Phys. 23 (1986), 57-65.

[19] D. Petz, Information-geometry of quantum states, in: Quantum Probability Communications, QP-PQ, X, World Sci. Publishing, 1998, 135-157.

[20] D. Petz and G. Tóth, The Bogoliubov inner product in quantum statistics, Lett. Math. Phys. 27 (1993), 205-216.

[21] C. R. Rao, Information and accuracy attainable in the estimation of statistical parameters, Rev. Bull. Calcutta Math. Soc. 37 (1945), 81-91.

[22] R. F. Streater Classical and quantum info-manifolds, Súrikaisekikenkyúsho Kókyúroku 1196 (2001), 32-51.

[23] F. Topsoe, Some inequalities for information divergence and related measures of discrimination, Res. Rep. Coll., RGMIA 2 (1999), 85-98.

[24] H. Umegaki, Conditional expectation in an operator algebra. IV. Entropy and information, Kōdai Math. Sem. Rep. 14 (1962), 59-85.

[25] H. Vermeil, Notiz über das mittlere Krümmungsmass einer n-fach ausgedehnten Riemann'schen Mannigfaltigkeit, Akad. Wiss. Göttingen Nachr. (1917), 334-344. 